\title{
Matrix Pre-Coated MALDI MS Targets for Small Molecule Imaging in Tissues
}

\author{
Kerri J. Grove, Sara L. Frappier, Richard M. Caprioli \\ Departments of Chemistry, Biochemistry, and the Mass Spectrometry Research Center, Vanderbilt University, MRB3, 465 \\ 21st Ave S, Nashville, TN 37235, USA
}

\begin{abstract}
A new sample preparation method for MALDI tissue imaging has been developed for the analysis of low molecular weight compounds that employs matrix pre-coated MALDI targets. Tissue sections need only to be transferred onto the pre-coated target before analysis for fast and easy sample preparation. Pre-coated targets have a homogenous matrix coating with uniform crystals of approximately $1-2 \mu \mathrm{m}$ and do not require solvents that may lead to analyte delocalization within a tissue section. We report here the use of matrix pre-coated targets for imaging of lipids, peptides, and pharmaceuticals in tissues.
\end{abstract}

Key words: MALDI imaging, Matrix application methods, Small molecule, Lipids, Peptides

\section{Introduction}

$\mathrm{M}$ atrix assisted laser desorption/ionization imaging mass spectrometry (MALDI IMS) can be used to generate molecular maps of tissue sections for proteins, peptides $[1,2]$, lipids [3, 4], pharmaceuticals, and metabolites [5]. Sample preparation is an important step in the MALDI IMS experiment that normally requires application of a MALDI matrix on top of the tissue section. Current matrix application methods include spray coating, microspotting [6], sublimation [3], and dry-coating [4] techniques. Spray coating and microspotting require the application of a solution of the matrix onto the tissue, producing matrix crystals after evaporation of the solvent. Although spray coating and microspotting have been used successfully, each of these techniques has certain drawbacks: spray coating is difficult to apply reproducibly and may lead to analyte delocalization if the target becomes too wet, microspotting requires costly equipment and has limited spatial resolution, and both of these require additional preparation time prior to the MS data acquisition. Dry coating and sublimation have advantages in producing uniformly small crystals across the sample and do not require solvents but are limited to the analysis of lipids and some other small molecules [7]. Previous work with pre-coated targets was reported for use with capillary electrophoresis separations followed by MALDI MS analysis where the capillary effluent

Correspondence to: Richard Caprioli; e-mail: r.caprioli@vanderbilt.edu was deposited on a matrix pre-coated membrane [8]. In addition, the use of a pre-coated target for imaging a drug compound in tissue where the tissue was blotted onto the matrix coated membrane surface has been reported [9].

We present a sample preparation procedure for MALDI imaging utilizing matrix pre-coated targets that is cost effective, fast, and essentially eliminates sample preparation steps following tissue sectioning. The purpose of this paper is to present our preliminary findings on the potential value of using matrix pre-coated targets and their utility for high-throughput applications. Tissue sections are simply mounted onto matrix pre-coated targets and then inserted directly into a mass spectrometer. Matrix pre-coated targets can be prepared in advance and stored in a dark environment for extended periods of time ( $>1$ month) to be used when needed for high-throughput studies. We demonstrate the use of pre-coated targets for imaging of lipids, peptides, and pharmaceuticals.

\section{Methods}

\section{Materials}

2,5-Dihydroxybenzoic acid (DHB) was purchased from Acros Organics and used without further purification. Whole rat brains and porcine pituitaries were purchased from PelFreeze Biologicals. Gold coated target plates were purchased from Applied Biosystems.

Received: 1 September 2010

Revised: 7 October 2010

Accepted: 11 October 2010

Published Online: 20 January 2011 


\section{Sample Target Preparation}

MALDI target plates were pre-coated with matrix by vapor deposition onto a clean target plate. Sublimation of the matrix was achieved with reduced pressure and heat. Approximately $300 \mathrm{mg}$ of DHB was placed in the bottom of the sublimation apparatus and a clean plate was fixed onto the bottom of the condenser above the matrix. The bottom of the apparatus was heated to $110^{\circ} \mathrm{C}$ for $7 \mathrm{~min}$ and had a pressure of 30-100 mtorr. Both gold targets and glass slides were used to make pre-coated targets.

\section{Evaluation of Matrix Surface}

DHB coated MALDI targets were analyzed with a scanning electron microscope (SEM) (S-4200, Hitachi). Scanning electron microscopy (SEM) was performed on samples as prepared (no conductive coating was applied) with an accelerating voltage of $2 \mathrm{kV}$. Morphology and size distribution were examined. The matrix layer thickness was determined by using a laser to completely ablate the matrix to the gold surface of the precoated target plate. The crater depth was then measured by using an Olympus BX50 microscope with the 3D Constructor module in the Image-Pro Plus Version 7 software.

\section{Intracerebral Tumor Model}

Six-week-old, female C57BL/6NCrl mice were anesthetized with $100 \mathrm{mg} / \mathrm{kg}$ ketamine and $10 \mathrm{mg} / \mathrm{kg}$ xylazine. Mice were immobilized in a stereotactic frame, an incision was made at the midline of the scalp, and a 0.5 -mm burr hole was drilled in the right forebrain, approximately $3 \mathrm{~mm}$ lateral from the midline and $1 \mathrm{~mm}$ anterior to bregma. A suspension of 180,000 GL26 murine cancer cells in phosphate buffered saline (PBS) was injected $3 \mathrm{~mm}$ deep (from the skull surface) into the burr hole. Incisions were closed with surgical staples. All techniques were performed by using sterilized instruments and the wound was created and closed by using aseptic procedures. Tumor growth was allowed to occur over a period of 15 days. The mice received $3 \mathrm{mg}$ of imatinib in $300 \mu \mathrm{L}_{\text {of }} \mathrm{H}_{2} \mathrm{O}$ at $\mathrm{pH}$ 5 , with control mice receiving $300 \mu \mathrm{L}$ of only the vehicle by esophageal gavage. Two hours after the single dose, each animal was put under anesthesia $(110 \mathrm{mg} / \mathrm{kg}$ ketamine and $10 \mathrm{mg} / \mathrm{kg}$ xylazine $)$ via intraperitoneal injection as a single dose followed by decapitation. Brains were excised and frozen on powdered dry ice.

\section{Tissue Preparation}

Tissues were stored at $-80{ }^{\circ} \mathrm{C}$ until use and then warmed to $-20{ }^{\circ} \mathrm{C}$ for sectioning with a Leica CM3050 S cryostat. Sections were cut at $8 \mu \mathrm{m}$. Sections following the pre-coated target protocol were thawmounted directly on the matrix pre-coated target plate and stored in a desiccator until analysis. For sections to be coated with matrix after cutting, the tissue was thaw-mounted on a clean target plate and stored in a desiccator until matrix application. Sections used for imaging were sublimated with DHB as described above. Sections used for lipid identifications were spotted with DHB at a concentration of $25 \mathrm{mg} / \mathrm{mL}$ in 50:50 ethanol/water with $100 \mathrm{mM}$
$\mathrm{LiCl}$. Sections used for peptide identifications were washed for $30 \mathrm{~s}$ in ethanol, dried, and then spotted with DHB at a concentration of $30 \mathrm{mg} / \mathrm{mL}$ in $50: 50$ acetonitrile/water containing $0.1 \%$ trifluoroacetic acid.

\section{Mass Spectrometry and Data Analysis}

MALDITOF MS analyses of lipids and peptides were acquired on a Bruker ultrafleXtreme in positive ion reflectron mode using the flexControl 3.3 software package. Analysis was performed with 200-500 shots/spectra with a $1 \mathrm{kHz}$ smartbeam II Nd:YAG laser and the laser intensity was adjusted before each imaging experiment. The samples on the pre-coated targets generally needed increased laser fluence. Image acquisition, normalization, and visualization were carried out using flexImaging 2.1 software and spectral analysis with flexAnalysis 3.3 software. Peptides were identified by using a timed ion gate for precursor ion selection, the fragments were further accelerated with $19 \mathrm{kV}$ in the LIFT cell, and detected after passage through the reflectron. Lipids were identified from tissue with MS/MS on a Thermo MALDI LTQ XL mass spectrometer in positive ion mode. Drug images were acquired by using a Thermo MALDI LTQ XL where the selected reaction monitoring (SRM) transition of imatinib $(\mathrm{m} / \mathrm{z} 494 \rightarrow 394)$ was followed. Data analysis was performed with ImageQuest software.

\section{Results and Discussion}

Previous work [10] mapped lipid distributions in tissue sections where the matrix was applied on top of tissues by sublimation. The homogenous fine crystal coating produced by sublimation provides excellent sensitivity and is applicable to high spatial resolution imaging. Applying DHB by sublimation, one obtains crystals that are 1-2 $\mu \mathrm{m}$ in size as seen in Figure 1a. The pre-coated matrix layer contains $\sim 0.25 \mathrm{mg} \mathrm{DHB} / \mathrm{cm}^{2}$ with a thickness of approximately $4 \mu \mathrm{m}$ that is uniform across the surface. In comparison, using standard dried droplet matrix deposition of DHB on the same target, one obtains crystals tens of microns in length with an average matrix coverage of $\sim 2 \mathrm{mg} / \mathrm{cm}^{2}$. To demonstrate the feasibility of matrix pre-coated targets for tissue imaging, a comparative lipid analysis from a post (top) coating matrix application and a pre-coated target was performed. Tissue sections up to $8 \mu \mathrm{m}$ were found to afford robust spectra when mounted on a matrix pre-coated target, whereas thicker sections tended to have reduced ion yield. Serial sagittal sections of a rat brain were cut, one mounted on a DHB pre-coated target and the other on a gold plate subsequently coated with DHB by sublimation. Figure $1 \mathrm{~b}-\mathrm{d}$ displays the results of this comparative analysis. The pre-coated target was shown to produce an image of essentially equal quality to that when matrix was sublimed on top of the tissue for lipid analysis when performed at a $100-\mu \mathrm{m}$ raster. It is noted that the relative intensity of several specific signals differed between the two methods. The basis for this is not currently fully understood and may be the result of different ionization efficiencies, although it is noted that these are two different tissue sections that would not be expected to give identical results.

We also sought to determine the detection capabilities of precoated targets for other small molecules. A porcine pituitary tissue 

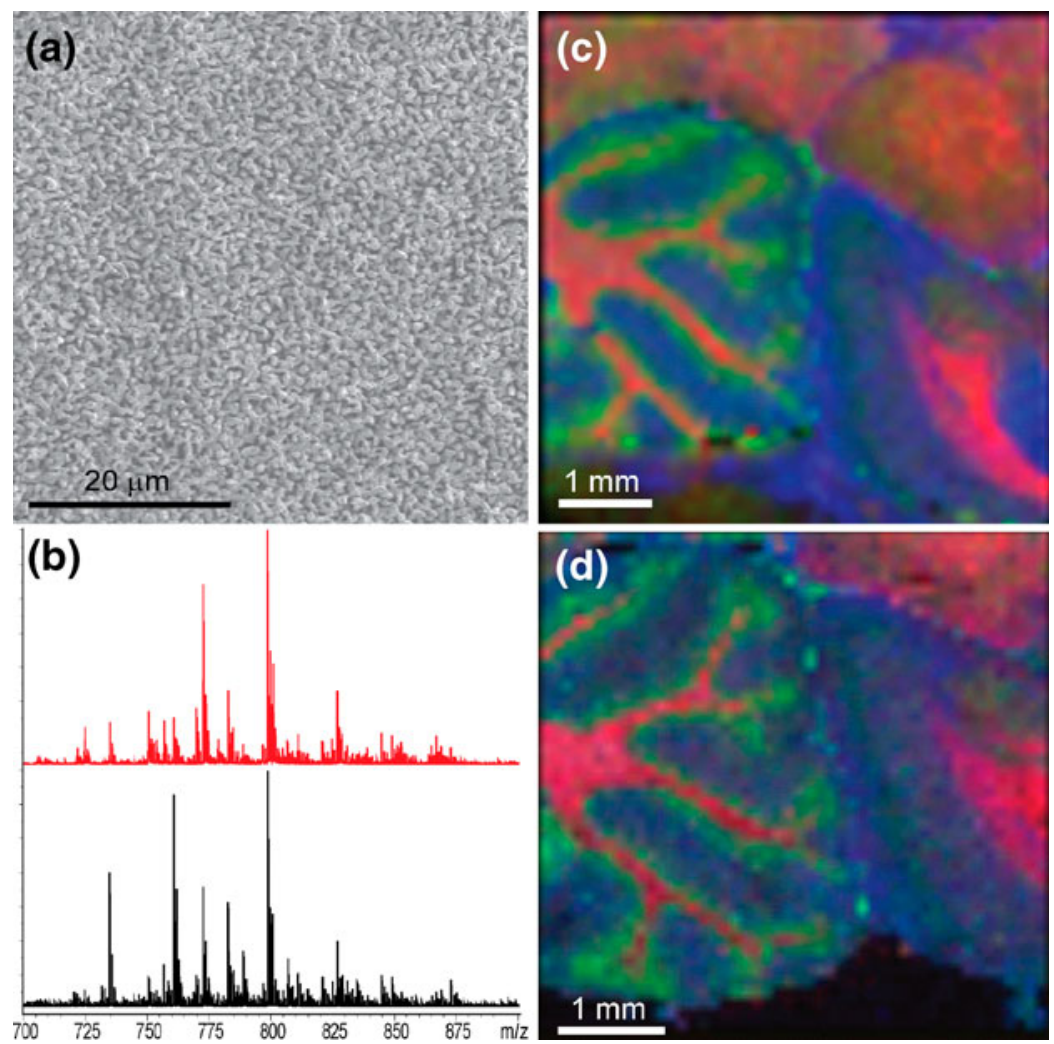

Figure 1. (a) SEM micrograph of DHB matrix crystals after sublimation onto a MALDI target. Scale bar $=20 \mu \mathrm{m}$. (b) Sum spectra from an image of a rat brain sagittal section prepared on a matrix pre-coated metal target (red) and a standard metal target that was sublimated with DHB after sectioning (black). Corresponding MALDI MS images of the brain sections prepared on a (c) matrix pre-coated target and (d) matrix post-coated target, i.e., matrix sublimated with DHB on top of tissue section. Colors correspond to ions of $\mathrm{m} / \mathrm{z} 769.5$ (SM 18:0, blue), $\mathrm{m} / \mathrm{z} 796.5$ (PC 34:2, green), and $\mathrm{m} / \mathrm{z} 826.5$ (PC $36: 1$, red). Images acquired at a 100- $\mu \mathrm{m}$ raster. Lipid identifications were performed by MS/MS experiments. Scale bars $=1 \mathrm{~mm}$
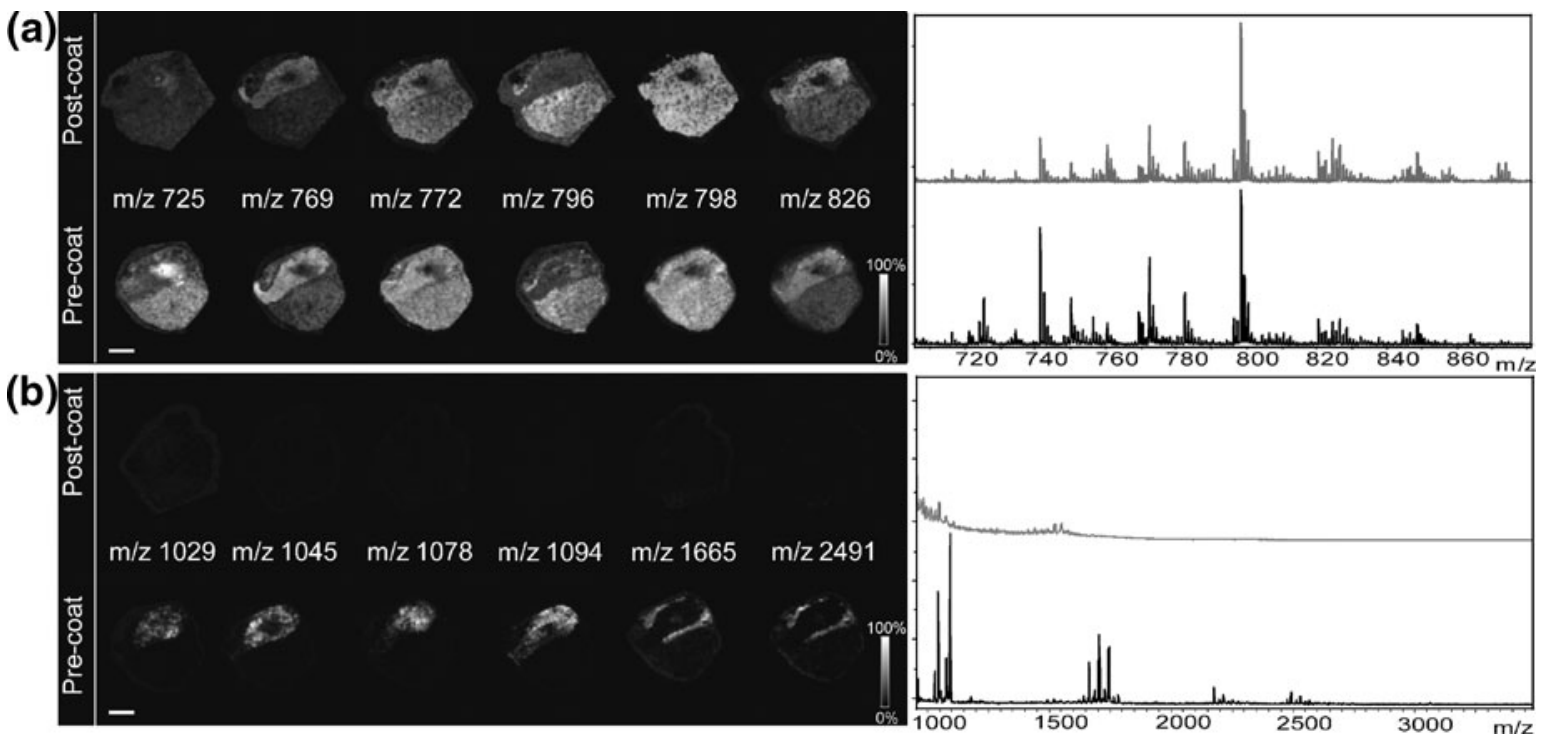

Figure 2. Comparison of images obtained from pituitary tissue on a DHB pre-coated target and a serial section with normal post-coating procedure each acquired with a 150- $\mu$ m raster. (a) Lipid images with sum spectra shown at right. (b) Peptide images from the same tissue section with spectra shown at right. No peptides were detected when matrix was applied by sublimation only over the tissue. Scale bar $=2 \mathrm{~mm}$ 

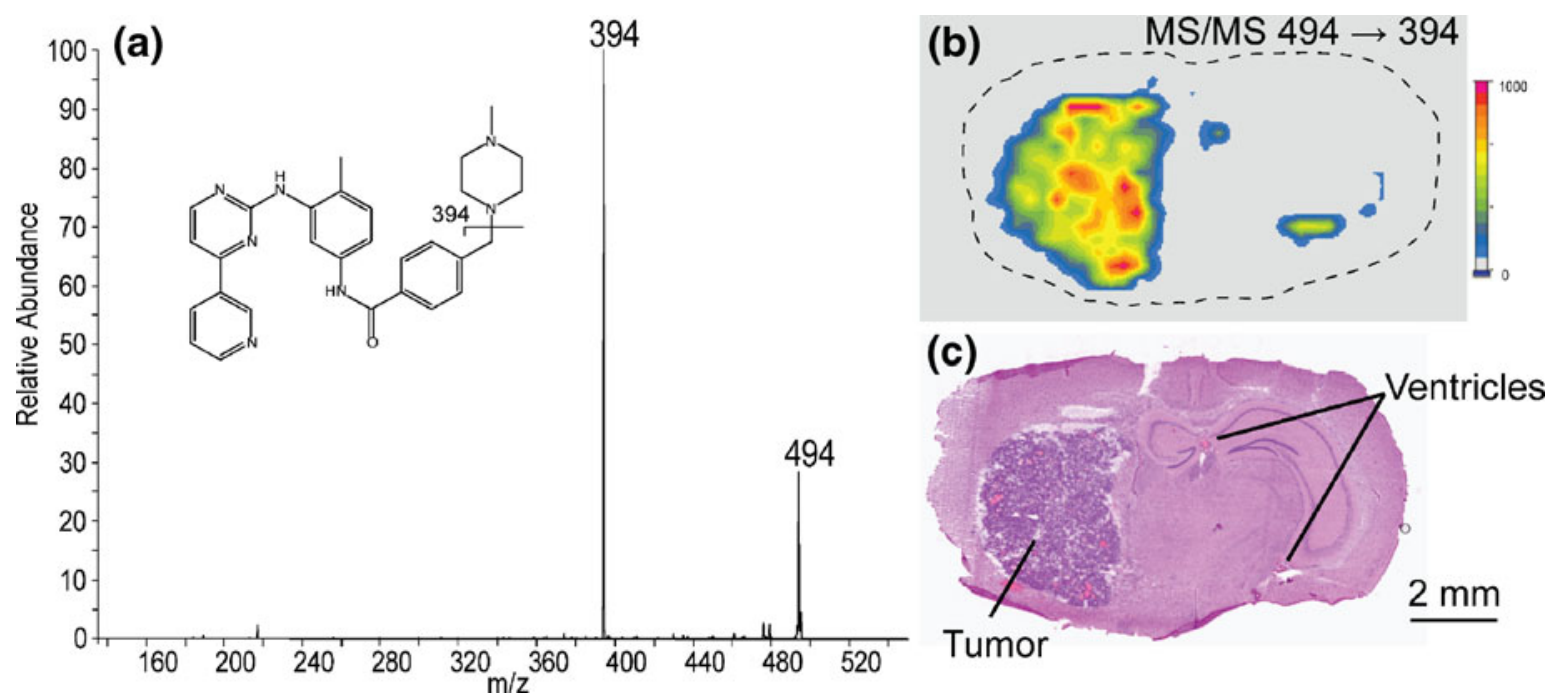

Figure 3. (a) MS/MS spectrum of imatinib ( $\mathrm{m} / \mathrm{z} 494)$. (b) The ion image of the $\mathrm{m} / \mathrm{z} 494 \rightarrow 394$ transition of the drug showing localization to the tumor and ventricles. Image acquired with a 300- $\mu$ m raster. (c) H\&E stained section showing the tumor and ventricles

section was mounted on a matrix pre-coated target and following laser ablation, peptide signals were recorded with markedly higher signal-to-noise than sublimation alone on top of a tissue section. Figure 2 compares identical DHB deposition methods for lipid and peptide imaging. Matrix pre-coating facilitates the detection of both peptides and lipids from the same tissue section, whereas sublimation of matrix on top essentially allowed only the detection of lipids. A possible reason for the increase in sensitivity is that because the tissue is thaw-mounted on a matrix pre-coated target, there is a greater degree of mixture of analytes with matrix.

The use of pre-coated targets for drug imaging studies has also been investigated. Imatinib is used in the treatment of cancer and was shown to localize to tumor regions in mouse brain [11]. The MS/MS analysis of the protonated imatinib molecular ion at $\mathrm{m} / \mathrm{z} 494$ gave a fragment at $m / z 394.1$ corresponding to the loss of methylpiperazine. We monitored this transition by tandem mass spectrometry and imaged a section of a brain with glioma from a mouse dosed with imatinib. The localization of the drug in the tumor is clearly seen in Figure 3. The two small regions indicating drug to the right of the tumor in the ion image correspond to the dorsal third ventricle and the lateral ventricle in the brain where blood flow occurs and where imatinib would also be expected. The tumor and vessels can be seen with an H\&E stain of a serial section of the brain. No background signal was detected in the gliomas of the undosed control mice.

This study shows that matrix pre-coated targets can be used to generate MALDI MS images directly from tissue for a variety of small molecules including lipids, peptides, and pharmaceutical compounds. Pre-coated targets offer a fast and reproducible matrix application for thin tissue section analysis and have the additional benefit of providing a homogenous and uniformly small crystal layer. Complete surface coverage of the matrix and elimination of solvents that can lead to analyte delocalization make this protocol suitable for high spatial resolution imaging applications. It also obviates the need for specialized matrix application equipment and the requisite expertise needed, rendering imaging MS more readily accessible to the average user for the analysis of low molecular weight analytes.

\section{Acknowledgments}

The authors would like to thank Peggi Angel for assistance with lipid identification and Kristina Schwamborn for assistance with peptide identification. SEM analysis was performed through the use of the VUMC Cell Imaging Shared Resource. The authors are grateful for the funding from NIH/NIGMS grant 5RO1GM058008-11 and DOD grant W81XWH-05-1-0179.

\section{References}

1. Caprioli, R.M., Farmer, T.B., Gile, J.: Molecular imaging of biological samples: localization of peptides and proteins using MALDI-TOF MS. Anal. Chem. 69(23), 4751-4760 (1997)

2. Chaurand, P., Caprioli, R.M.: Direct profiling and imaging of peptides and proteins from mammalian cells and tissue sections by mass spectrometry. Electrophoresis 23(18), 3125-3135 (2002)

3. Hankin, J.A., Barkley, R.M., Murphy, R.C.: Sublimation as a method of matrix application for mass spectrometric imaging. J. Am. Soc. Mass Spectrom. 18(9), 1646-1652 (2007)

4. Puolitaival, S.M., Burnum, K.E., Cornett, D.S., Caprioli, R.M.: Solventfree matrix dry-coating for MALDI imaging of phospholipids. J. Am. Soc. Mass Spectrom. 19(6), 882-886 (2008)

5. Khatib-Shahidi, S., Andersson, M., Herman, J.L., Gillespie, T.A., Caprioli, R.M.: Direct molecular analysis of whole-body animal tissue sections by imaging MALDI mass spectrometry. Anal. Chem. 78(18), 6448-6456 (2006)

6. Aerni,H.-R., Cornett,D.S., Caprioli, R.M.: Automated acoustic matrix deposition for MALDI sample preparation. Anal. Chem. 78(3), 827-834 (2005)

7. Goodwin, R.J.A., Scullion, P., MacIntyre, L., Watson, D.G., Pitt, A.R.: Use of a solvent-free dry matrix coating for quantitative matrix-assisted laser desorption ionization imaging of 4-bromophenyl-1,4-diazabicyclo(3.2.2)nonane-4-carboxylate in rat brain and quantitative analysis of the drug from laser microdissected tissue regions. Anal. Chem. 82(9), 3868-3873 (2010)

8. Zhang, H., Caprioli, R.M.: Capillary electrophoresis combined with matrix-assisted laser desorption/ionization mass spectrometry; continuous sample deposition on a matrix-precoated membrane target. J. Mass Spectrom. 31(9), 1039-1046 (1996)

9. Bunch, J., Clench, M.R., Richards, D.S.: Determination of pharmaceutical compounds in skin by imaging matrix-assisted laser desorption/ ionisation mass spectrometry. Rapid Commun. Mass Spectrom. 18(24), 3051-3060 (2004)

10. Murphy, R.C., Hankin, J.A., Barkley, R.M.: Imaging of lipid species by MALDI mass spectrometry. J. Lipid Res. 50(Supplement), S317-S322 (2009)

11. Cornett, D.S., Frappier, S.L., Caprioli, R.M.: MALDI-FTICR imaging mass spectrometry of drugs and metabolites in tissue. Anal. Chem. 80 (14), 5648-5653 (2008) 